\title{
Improvement of Lesion Phenotyping in Cercospora beticola-Sugar Beet Interaction by Hyperspectral Imaging
}

\author{
Marlene Leucker, Anne-Katrin Mahlein, Ulrike Steiner, and Erich-Christian Oerke
}

Institute for Crop Science and Resource Conservation (INRES)-Phytomedicine, University of Bonn, Meckenheimer Allee $166 \mathrm{a}$, 53115 Bonn, Germany.

Accepted for publication 18 October 2015.

\begin{abstract}
Leucker, M., Mahlein, A.-K., Steiner, U., and Oerke, E.-C. 2016. Improvement of lesion phenotyping in Cercospora beticola-sugar beet interaction by hyperspectral imaging. Phytopathology 106:177-184.

Cercospora leaf spot (CLS) caused by Cercospora beticola is the most destructive leaf disease of sugar beet and may cause high losses in yield and quality. Breeding and cultivation of disease-resistant varieties is an important strategy to control this economically relevant plant disease. Reliable and robust resistance parameters are required to promote breeding progress. CLS lesions on five different sugar beet genotypes incubated under controlled conditions were analyzed for phenotypic differences related to field resistance to $C$. beticola. Lesions of CLS were rated by classical quantitative and qualitative methods in

combination with noninvasive hyperspectral imaging. Calculating the ratio of lesion center to lesion margin, four CLS phenotypes were identified that vary in size and spatial composition. Lesions could be differentiated into subareas based on their spectral characteristics in the range of 400 to $900 \mathrm{~nm}$. Sugar beet genotypes with lower disease severity typically had lesions with smaller centers compared with highly susceptible genotypes. Accordingly, the number of conidia per diseased leaf area on resistant plants was lower. The assessment of lesion phenotypes by hyperspectral imaging with regard to sporulation may be an appropriate method to identify subtle differences in disease resistance. The spectral and spatial analysis of the lesions has the potential to improve the screening process in breeding for CLS resistance.
\end{abstract}

Cercospora leaf spot (CLS), caused by Cercospora beticola Sacc. an asexual fungus in the Ascomycota, is the most destructive leaf disease of sugar beets (Holtschulte 2000) and may cause yield losses of up to $43 \%$ (Shane and Teng 1992). Warm and humid climate conditions are disease-promoting (Bleiholder and Weltzien 1972; Shane and Teng 1992). Application of fungicides is the common strategy to control CLS; however, resistance to strobilurins and benzimidazoles has been reported for $C$. beticola (Karaoglanidis et al. 2000; Kirk et al. 2012; Trkulja et al. 2012). Hence, cultivation of disease-resistant sugar beets is essential for sustainable disease management.

Infected leaves exhibit reddish-brown lesions with a white to grayish center in which black pseudostromata are formed. In later stages, lesions coalesce and may cause complete collapse of the leaves. Detailed microscopic studies have been conducted to reveal processes during pathogenesis. C. beticola enters the host through stomata and colonizes the leaf tissue intercellularly (Feindt et al. 1981; Steinkamp et al. 1979). The pathogen produces toxins such as cercosporin (Daub and Ehrenshaft 2000) and beticolins (Goudet et al. 2000) in order to necrotize plant cells and make nutrients available. This leads to an almost simultaneous collapse of the cells in the infected area. The hyphae grow densely and form pseudostromata below stomata. Melanized conidiophores emerge in the necrotic center of the lesion and produce long, acicular conidia that may be spread by wind or water to infect new leaves or plants (Weiland and Koch 2004). Quantitative resistance to C. beticola has been described as rate limiting (Rossi et al. 1999). It is characterized by a reduced infection efficiency of conidia, longer incubation time, reduced spore production, and smaller lesions. Furthermore, it is assumed that leaf age and stomatal

Corresponding author: M. Leucker; E-mail address: mleucker@uni-bonn.de

http://dx.doi.org/10.1094/PHYTO-04-15-0100-R

(C) 2016 The American Phytopathological Society opening influence disease severity (Feindt et al. 1981). However, resistance mechanisms are still unclear. Often, existing cultivars with higher resistance have a lower root yield potential under nonstress conditions compared with conventional, susceptible cultivars (Smith and Campbell 1996). Therefore, there is a high demand for genotypes that combine effective resistance and high productivity.

In order to promote progress in resistance breeding, reliable and precise estimates of disease severity are important (Montes et al. 2007; Bock et al. 2010). Examining symptom phenotypes by hyperspectral imaging is a promising tool to characterize and to understand important aspects of plant-pathogen interactions (Mutka and Bart 2015; Kuska et al. 2015). With this sensor-based technique, electromagnetic spectra for all pixels of an image are recorded. Spectral properties of plants are influenced by the morphology and the biochemical composition of plant tissue. The strong absorption in the visible range (VIS, 400 to $700 \mathrm{~nm}$ ) due to pigments is characteristic for leaf reflection of sunlight (Jensen 2007). An increased reflection in the near-infrared range (NIR, 700 to $1,100 \mathrm{~nm}$ ) results from multiple scattering processes, depending on the leaf surface and the internal cell structure. Plant compounds and water influence the light absorption in the shortwave infrared (SWIR, 1,100 to 2,500 nm) (Jacquemoud and Ustin 2001). The interaction between the plant and a pathogen leads to structural and metabolic modifications which change the spectral characteristics. These effects on leaf reflectance provide the basis for spectral investigations on disease phenotypes. Hyperspectral imaging enables the detection, identification and quantification of plant diseases (Mahlein et al. 2012). It was demonstrated that the three foliar diseases (CLS, powdery mildew, and sugar beet rust) had significantly different spectral signatures. Because these signatures were disease-specific, a clear identification was possible (Mahlein et al. 2010). Diseased leaves could be quantified by the spectral angle mapper (SAM) classification (Mahlein et al. 2012). Furthermore, a pixel-wise 
extraction of spectral reflectance enabled a pathogenesis-related differentiation of CLS lesions into subareas.

Examining symptom phenotypes may provide new insights into how pathogens influence host physiology and how plants respond to pathogen infection. CLS lesions on five sugar beet genotypes with varying disease susceptibility were analyzed by hyperspectral imaging in order to improve phenotyping of CLS resistance in sugar beet breeding. Microscopic investigations on various scales and the quantification of the sporulation were used to characterize CLS lesions for reference.

\section{MATERIALS AND METHODS}

Plant material. Five homozygous sugar beet breeding lines, Bv1, Bv2, Bv4, Bv6, and Bv7 (KWS SAAT SE, Einbeck, Germany), with different susceptibility to $C$. beticola were chosen for the experiments. Differences in susceptibility have been assessed in multi-year field trials by the breeders (W. Beyer, personal communication). Seeds were pregrown in small pots until growth stage BBCH 12 (Meier 2001). Seedlings were transferred into commercial substrate (Einheitserde ED73, Klasmann-Deilmann, Geeste, Germany) in plastic pots $(\varnothing 13 \mathrm{~cm})$. Plants were cultivated under controlled conditions at $22^{\circ} \mathrm{C}$ day and $20^{\circ} \mathrm{C}$ night, $60 \pm 10 \%$ relative humidity (RH), and a photoperiod of $16 \mathrm{~h}$. Plants were watered as necessary and protected against powdery mildew by regular application of sublimate sulfur. Six weeks after transplanting, the sugar beets were fertilized weekly with a $0.2 \%$ solution of Poly Crescal (Aglukon GmbH, Duesseldorf, Germany). Eight- to nine-week-old plants were used for the experiments after washing off the sulfur.

Pathogen and inoculation. Inoculum of $C$. beticola was harvested from heavily diseased sugar beet leaves that had been sampled from field crops in late summer. The dry leaves were incubated at $100 \% \mathrm{RH}$ for $48 \mathrm{~h}$ in order to induce sporulation of the pathogen. Conidia were washed off with a $0.01 \%$ solution of Tween 20 (Merck Millipore, Darmstadt, Germany) and a conidia suspension $\left(4 \times 10^{4}\right.$ conidia $\left.\mathrm{ml}^{-1}\right)$ was sprayed onto sugar beet leaves using a hand sprayer. Inoculated plants were incubated at $100 \% \mathrm{RH}$ and $25 / 20^{\circ} \mathrm{C}$ for $48 \mathrm{~h}$ and then placed back to $60 \pm 10 \%$ $\mathrm{RH}$. At least three independent inoculation trials, each with a minimum of four plants per genotype were conducted. Four fully grown leaves per plant were used for investigations.

Disease assessment. Disease severity was assessed by visual rating of the percentage of diseased leaf area of two fully developed leaf pairs. In addition, the number of lesions per leaf was counted every second day throughout the experiment ( $n \geq 12$ leaves). Lesions were examined using a stereo microscope (MZ16 F, Leica Microsystems, Wetzlar, Germany). Images were taken with a mounted digital camera (KY-F75U, JVC, Yokohama, Japan). To investigate the growth rate of CLS, the size of at least 10 developing lesions was measured every day using the software Discus 4.6 (Technisches Buero Hilgers, Koenigswinter, Germany). The size of approximately 100 mature lesions on each genotype was measured to assess different phenotypes. When visible, lesion centers were also measured and the size of the darker margin was calculated as difference between total lesion area and central area according to equation 1 .

$$
\operatorname{Margin}\left(m m^{2}\right)=\operatorname{Lesion}\left(m m^{2}\right)-\text { Center }\left(m m^{2}\right)
$$

The different lesion phenotypes were categorized based on the calculated center to margin ratio (equation 2 ).

$$
\text { Ratio }=\frac{\text { Center }\left(\mathrm{mm}^{2}\right)}{\operatorname{Margin}\left(\mathrm{mm}^{2}\right)}
$$

Measurement of spore production. Spore production was measured 4 weeks after inoculation according to Karadimos et al.
(2005). Sporulation was induced by incubating diseased plants at $100 \% \mathrm{RH}$ for $48 \mathrm{~h}$. Leaf disks ( $\varnothing 11 \mathrm{~mm})$ with a single lesion each were placed into $0.5 \mathrm{ml}$ of tap water with $0.01 \%$ Tween 20 each and vortexed to detach spores from conidiophores. Then, spores were counted using a Fuchs-Rosenthal chamber and the corresponding lesion size was measured using a stereo microscope as described above. Twenty lesions per genotype were analyzed and results expressed as the number of conidia per square millimeter of diseased leaf area.

Hyperspectral measurements and image analysis. Spectral reflectance was measured with a spectral line scanner (Spectral camera PFD V10E, Spectral Imaging Ltd., Oulu, Finland) mounted on a zoom system (Z6 APO, Leica, Germany) with a XY-motorized stage (Prior Scientific GmbH, Jena, Germany). Two lightlines (Schott AG, Mainz, Germany) were used for homogenous illumination. Hyperspectral images were recorded using the software SpectralCube (Spectral Imaging Ltd.) Two plants representing the typical infection situation (= average disease incidence and severity) of the sugar beet lines and two to four leaves per plant were used per genotype and treatment for measurements on the adaxial leaf surface. At least 10 hyperspectral images with CLS lesions were analyzed per genotype.

The reflectance of samples was calculated by normalizing the images relative to the reflection of a $100 \%$ white reference standard (Zenith Polymer Target, SphereOptics GmbH, Uhldingen, Germany) and to a dark current measurement of the inherent irradiance of the sensor after closing a shutter using the software ENVI 5.1 + IDL 8.3 (ITT Visual Information Solutions, Boulder, CO). Before analysis, spectral signals were smoothed by applying the Savitzky-Golay filter (Savitzky and Golay 1964).

The supervised classification of the hyperspectral images allows a categorization of single image pixels. The image element is assigned to one out of four defined classes based on the similarity to the distinct reference spectra (endmembers) of the respective class. The spectral angle mapping method (SAM, Yuhas et al. 1992) was performed using the ENVI 5.1 + IDL 8.3 software. For CLS classification, the categories center, transition area, margin, and noninfected were chosen. The classification converts the hyperspectral image into a pseudo-color image. Nonclassified pixels are represented in black.

Scanning electron microscopy. Scanning electron microscopic observations were obtained using a Phenom G2 Pure (Phenom-World, Eindhoven, Netherlands). Freshly harvested leaf disks with sporulating CLS lesions were gold coated using an automated sputter coater (MSC 1T, LOT Quantum Design, Darmstadt, Germany). Images of at least three lesions of the most predominant symptom type of each genotype were recorded.

Statistical evaluation. Statistical analysis was conducted using SPSS 22.0 (SPSS Inc., Chicago, IL). After significant differences were found, the means between the five genotypes were compared using Tukey's honestly significant difference (HSD) test with a significance level of $P=0.05$ confidence in order to separate subgroups. Experiments were repeated three times. The number of biological replicates per genotype $(n)$ is given for each experiment.

\section{RESULTS}

Progress of CLS disease on different sugar beet genotypes. Five sugar beet genotypes were selected based on their susceptibility to $C$. beticola. To confirm the assessment of the breeder, disease progress was evaluated in experiments under greenhouse conditions (Fig. 1). Genotypes Bv6 and Bv7 were highly susceptible. The first lesions appeared 9 days postinoculation (dpi) on genotype Bv7 and 10 dpi on all other genotypes. The diseased leaf area increased within 10 days up to 70 and $50 \%$ on Bv7 and Bv6, respectively (Fig. 1A). Disease was less severe on the other three genotypes. The genotypes Bv2 and Bv4 showed lower percent 
diseased leaf area over time with an increase up to approximately $20 \%$. Bv1 was the least infected genotype with only $10 \%$ diseased leaf area at $19 \mathrm{dpi}$. The number of CLS lesions per leaf increased on all genotypes continuously over the experimental time (Fig. 1B). Bv6 and Bv7 had about two times more lesions than Bv4 and even seven times more than Bv1 and Bv2. Typical lesions appeared as well-defined depressions of the leaf surface, but this was rarely observed on genotype Bv4; instead appearance was characterized by small dark spots. Lesions on Bv6 and Bv7 were approximately $4 \mathrm{~mm}^{2}$ in size and considerably larger than on Bv1, Bv2, and Bv4 at the day of first appearance (Fig. 1C). They grew by up to $2 \mathrm{~mm}^{2}$ within the first 3 days and then reached final sizes varying from 5 to $6 \mathrm{~mm}^{2}$ on susceptible genotypes and 3 to $4 \mathrm{~mm}^{2}$ on resistant sugar beets, respectively.

In order to investigate the spore density of $C$. beticola, the number of conidia per diseased leaf area was assessed after 2 days of incubation under $100 \%$ RH (Fig. 1D). With about 40 conidia per mm², the spore density was lowest on genotype $\mathrm{Bv} 4$, followed by that on Bv1 with 95 per $\mathrm{mm}^{2}$. Both genotypes were significantly different from the highly susceptible Bv6 and Bv7, on which $C$. beticola produced 260 and 370 conidia $/ \mathrm{mm}^{2}$, respectively $(P \leq 0.01)$. The spore density was intermediate on genotype Bv2.

Variation of lesion phenotypes on sugar beet genotypes. The typical CLS symptom is a circular, brown to red lesion with gray center. However, the variation in lesion appearance among and within genotypes was high. Alternatively, lesions had a wide margin and small center or a large, dominating center with a thin margin. Every constellation in between was possible. Furthermore, some lesions were uniform without visible segmentation into margin and center, and the color reached from white over gray to brown, with or without reddish or brown margin. The lesion variability was investigated in detail in order to know whether lesion types are related to genotypic disease resistance. The CLS phenotypes were categorized based on the ratio of center to margin (Fig. 2) and the frequency of lesions on the sugar beet genotypes was assessed (Fig. 3 ). On genotypes Bv1, Bv2, and Bv4, more than $50 \%$ of the lesions showed no differentiation in margin and center (class 0 ). About one third of the lesions had a smaller center than margin or central and marginal areas about the same size (class 1 ). Only very few leaf spots had a larger center than margin. The genotypes with higher susceptibility to CLS (Bv6 and Bv7) had a lower percentage of uniform lesions (13 and 18\%) and more lesions with a ratio of $>1$ to 2 . Roughly $15 \%$ of the lesions on Bv7 had a predominant center with a size more than double the margin size.

Spectral characterization of CLS lesions. Spectral reflectance of CLS lesions was analyzed in order to characterize how phenotypes depend on the host genotype. Average spectral signatures (from 400 to $900 \mathrm{~nm}$ ) of lesions typical for the genotypes were extracted and compared. Reflectance spectra of mature CLS lesions were generally characterized by an increased reflectance in the VIS range and a decrease in the NIR range compared with the signature of noninfected leaf tissue. Reflectance of healthy tissue could be averaged over the five genotypes because differences between cultivars were low (Fig. 4). The differences between the CLS spectra of the genotypes were small, only lesions on Bv4 differed especially in the red range of the spectrum.

In a further step, spectral signatures of specific subareas of the lesions were manually extracted. Interestingly, the subarea-specific reflectance spectra on the genotypes were remarkably similar (Fig. 5). Reflectance of the lesion margins was characterized by a decreased chlorophyll peak and an increased reflectance between 580 and $700 \mathrm{~nm}$ compared with spectra of noninfected tissue (Fig. 5A and B).
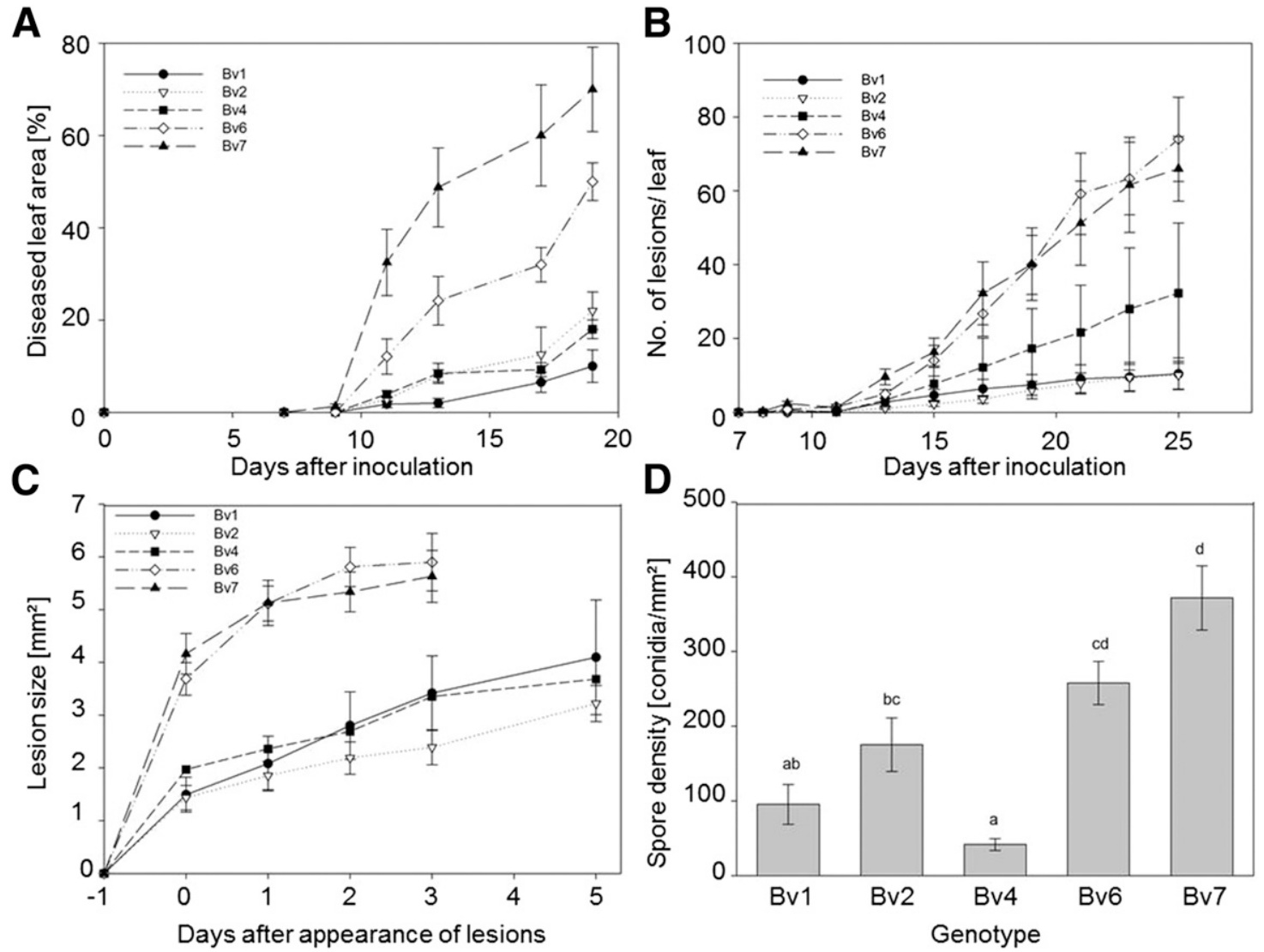

Fig. 1. Assessment of Cercospora leaf spot (CLS) on sugar beet genotypes Bv1, Bv2, Bv4, Bv6, and Bv7. A, Progress of leaf area covered with CLS within 3 weeks after inoculation ( $n=12$ leaves). B, Number of CLS lesions per leaf starting from the day of appearance of the first lesions 9 days after inoculation ( $n=16$ leaves). C, Size of developing CLS lesions within 3 days after their appearance on Bv1, Bv2, Bv6 and Bv7 ( $n=10$ lesions). D, Spore density as number of conidia per unit diseased leaf area after 2 days of incubation under $100 \%$ relative humidity on the five genotypes ( $n=20$ lesions); values with the same letter are not significantly different (Tukey's honestly significant different test, $P=0.05$ ). Bars indicate standard error of the mean. 
Additionally, the slope at the VIS-NIR transition (red edge) was less steep. Generally, reflectance of lesion centers was much higher in the VIS range than of the noninfected leaf tissue (Fig. 5D). It was also higher in the near-infrared range. With the spectral information from 400 to $900 \mathrm{~nm}$, it was possible to differentiate more explicit lesion subareas than only center and margin. A transition area between the margin and the center with an intermediate spectral signature could be identified (Fig. 5C). For Bv4, only two subareas could be detected. The comparison with the spectral signatures of the other genotypes indicated that spectral characteristics for the central area were absent on this genotype. Using the specific spectra as class reference spectra (endmembers), SAM classification was applied to the hyperspectral images (Fig. 6). As visualized by the pseudo-color images, composition of lesions varied with the sugar beet genotype. In accordance with the visual results (Fig. 3), the size of lesion centers was extended on Bv6 and $\mathrm{Bv} 7$ and the transition area was more pronounced on Bv1 and Bv2.

Topography of sporulating CLS lesions. Scanning electron micrographs of typical, sporulating CLS lesions on the different sugar beet genotypes are shown in Figure 7. The long, acicular spores were removed during sample preparation, but conidiophores protruding out of the leaf surface were visible. Segmentation of lesions was universal on all genotypes. Lesions were separated from uninfected tissue by a ring of shriveled tissue. This was adjacent to a transition area with sparsely emerging conidiophores. In the lesion centers, many mature conidiophores erupted from the tissue in small groups. The number of conidiophores and the size of the transition area and center varied among and within genotypes tending toward more conidiophores on the susceptible genotypes. Especially, the centers with mature conidiophores on Bv4 were much smaller than on the other genotypes (Fig. 7).

\section{DISCUSSION}

There are several ways to assess disease severity of CLS on sugar beets, but it is mostly rated based on the average percentage of

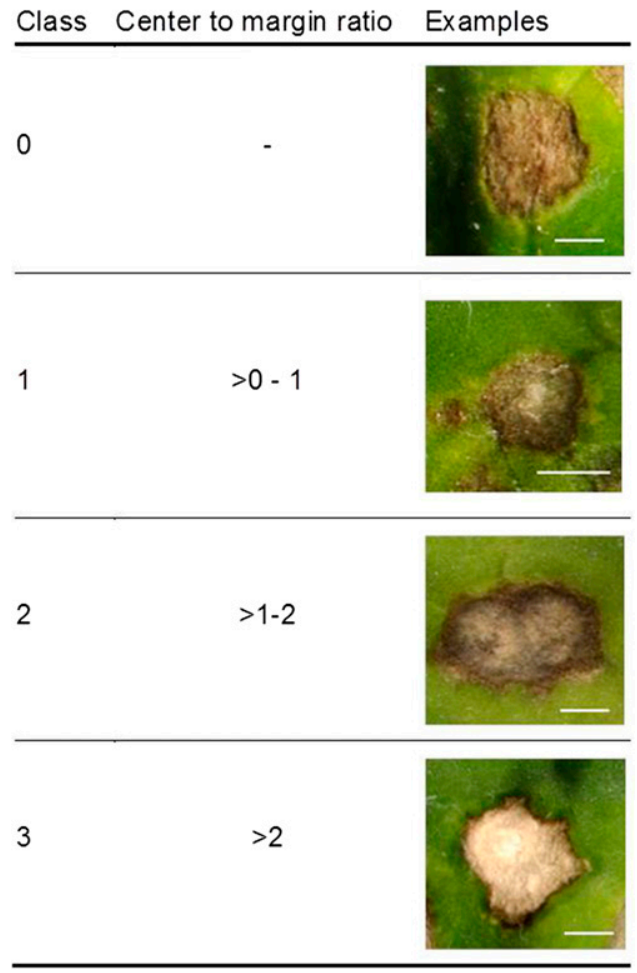

Fig. 2. Classes of Cercospora leaf spot lesions based on the ratio of center size to margin size. Exemplary lesions with corresponding center to margin ratio 21 days after inoculation. Scale bars represent $1 \mathrm{~mm}$. infected leaf area (Rossi et al. 1999; Wolf and Verreet 2002). Disease progress results from the combination of an increase in the number of lesions and in the size of leaf spots. The period of lesion growth was limited to a few days, whereas the increase in lesion number was observed for more than 2 weeks after the appearance of the first leaf spots. On most genotypes initial lesions appeared as well-defined depressions of the leaf surface and the initial lesion diameter was related to host plant resistance, indicating an inhibition of pathogen colonization. The host plant resistance had

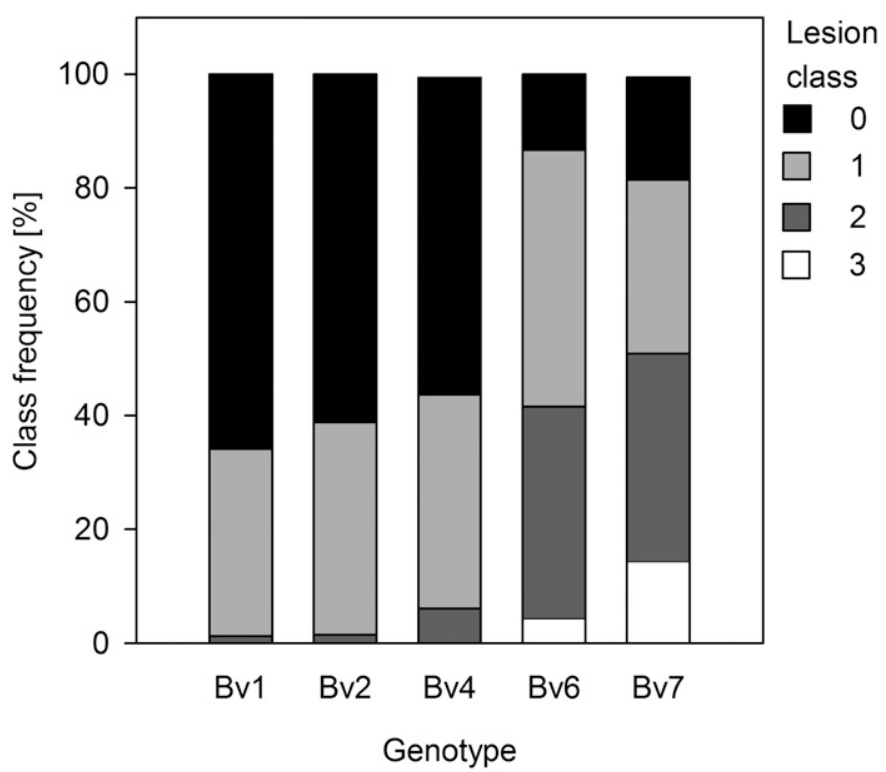

Fig. 3. Frequency of four Cercospora leaf spot classes based on the center to margin ratio on five sugar beet genotypes differing in Cercospora beticola susceptibility. The size of $\geq 100$ mature lesions on each genotype was measured 20 to 22 days after inoculation. When visible, lesion centers were measured, and the size of the darker margin was calculated as difference of total lesion area and central area. For details of lesion classes see Figure 2.

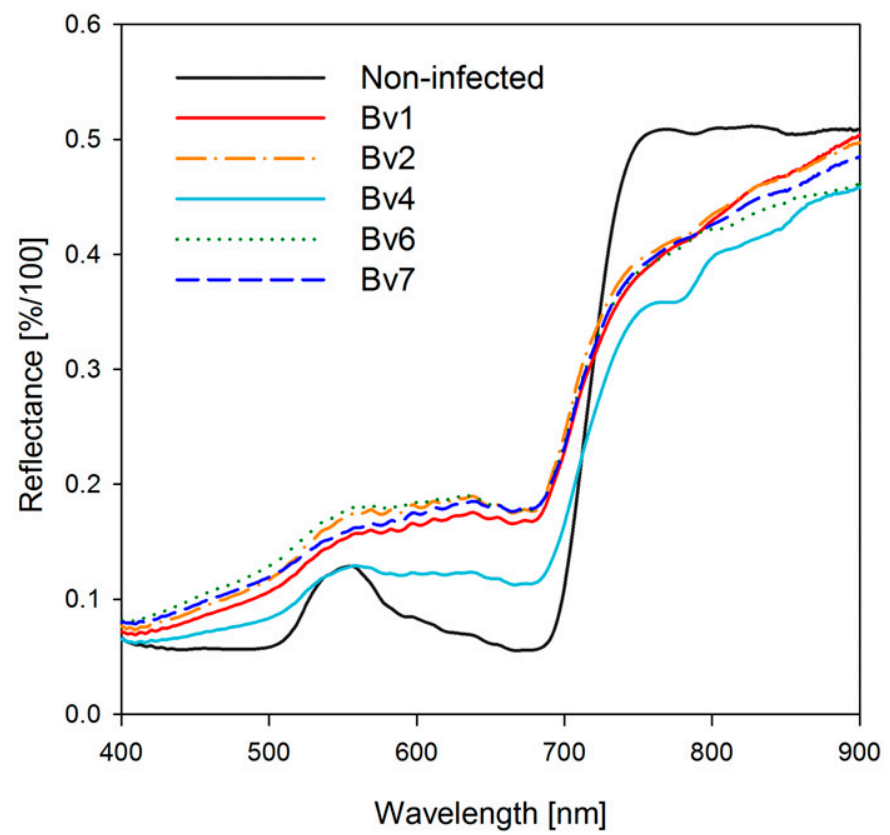

Fig. 4. Spectral signatures of noninfected tissue and Cercospora leaf spot (CLS) lesions on five different sugar beet genotypes varying in Cercospora beticola susceptibility. Reflectance spectra of noninfected tissue represent the mean of the five genotypes. CLS signatures are the mean of five lesions per genotype each. 
no significant effect on the time until appearance of the first lesion. Under controlled conditions, where no sporulation was induced, the increase of lesion numbers with time could not be due to a new infection cycle. Differences in the time until appearance of the lesions on the same genotype could result from a longer time until spore germination or delayed growth of some germ tubes (Feindt et al. 1980; Rossi et al. 2000). The lower infection rate of resistant sugar beet genotypes is in accordance with the report of Rossi et al. (1999). They also observed a reduced number of CLSs on resistant cultivars and significantly larger lesions on susceptible sugar beets. Furthermore, sporulation on a resistant genotype was reduced to $35 \%$ (Rossi et al. 2000). Similar host-pathogen relationships were observed in barley infected with Cochliobolus sativus. Fetch and Steffenson (1999) distinguished varying host-pathogen compatibility of spot blotch based on the type and relative size of lesions. Differences in components of rate-reducing resistance such as reduced sporulation were also described in the peanut-Cercospora arachidicola interaction (Ricker et al. 1985). Furthermore, Niks and Kuiper (1983) described reduced infection success accompanied by impaired growth and development of rust colonies on partially resistant barley. Infection types may also vary within categories from low to high even in qualitative resistance (of wheat and barley) to races of rust fungi (Kolmer 1996; Parlevliet 1976).

Analysis of symptom phenotypes has the potential to give insights into the physiology of the host-pathogen interactions (Mutka and Bart 2015). The sugar beet genotypes differing in resistance to $C$. beticola exhibited several CLS phenotypes per genotype. It was observed in all experiments that the lesions on the resistant genotypes had mostly smaller centers than those on the susceptible ones. This phenomenon is probably the consequence of the individual host-pathogen relationship. The result of the interaction is based on the genotype of both organisms. Ruppel (1972) characterized 14 C. beticola isolates which varied in morphology in vitro and virulence. However, differences in disease severity among sugar beet lines were highly significant in all tests and examined host resistance was effective against all isolates. The well described, race-specific resistance in sugar beet against the isolate C2 plays hardly any role in the field (Duffus and Ruppel 1993; Weiland and Koch 2004). Breeders aim at a broad-spectrum resistance against all populations of a pathogen under various environments. Numerous genes in pathogen recognition, cell signaling, and defense-related proteins, e.g., with chitinase or glucanase activity, are involved in quantitative resistance against C. beticola (Gottschalk et al. 1998; Nielsen et al. 1997; Weltmeier et al. 2011). In a transcription analysis, Weltmeier et al. (2011) found that polygenic (quantitative) resistance was characterized by a stronger defense response in the late stage (formation of necrotic lesions) than in the susceptible interaction.

The categorization of the different lesion types based on the ratio of center to margin and the frequency on the different genotypes implied a tendency from no lesion centers to larger centers on susceptible genotypes compared with resistant sugar beets. The larger centers might be associated with the significantly higher spore density per diseased leaf area on genotypes Bv6 and Bv7, because conidiophores are produced in this central area (Weiland and Koch 2004). The resistant genotype with the lowest spore density showed no lesion centers in the spectral analysis. Decreased spore production is one important component of quantitative resistance that delays disease epidemics as, for example, in barley cultivars resistant to Puccinia hordei and in peanuts resistant to C. arachidicola (Parlevliet 1979; Foster et al. 1980).

The spectral characterization of CLS revealed that the reflectance varied within the lesions. Four spectral signatures could be extracted along a transect from healthy green tissue to the center of the diseased tissue. These signatures differed from each other in explicit regions of the electromagnetic spectra. The increase in the
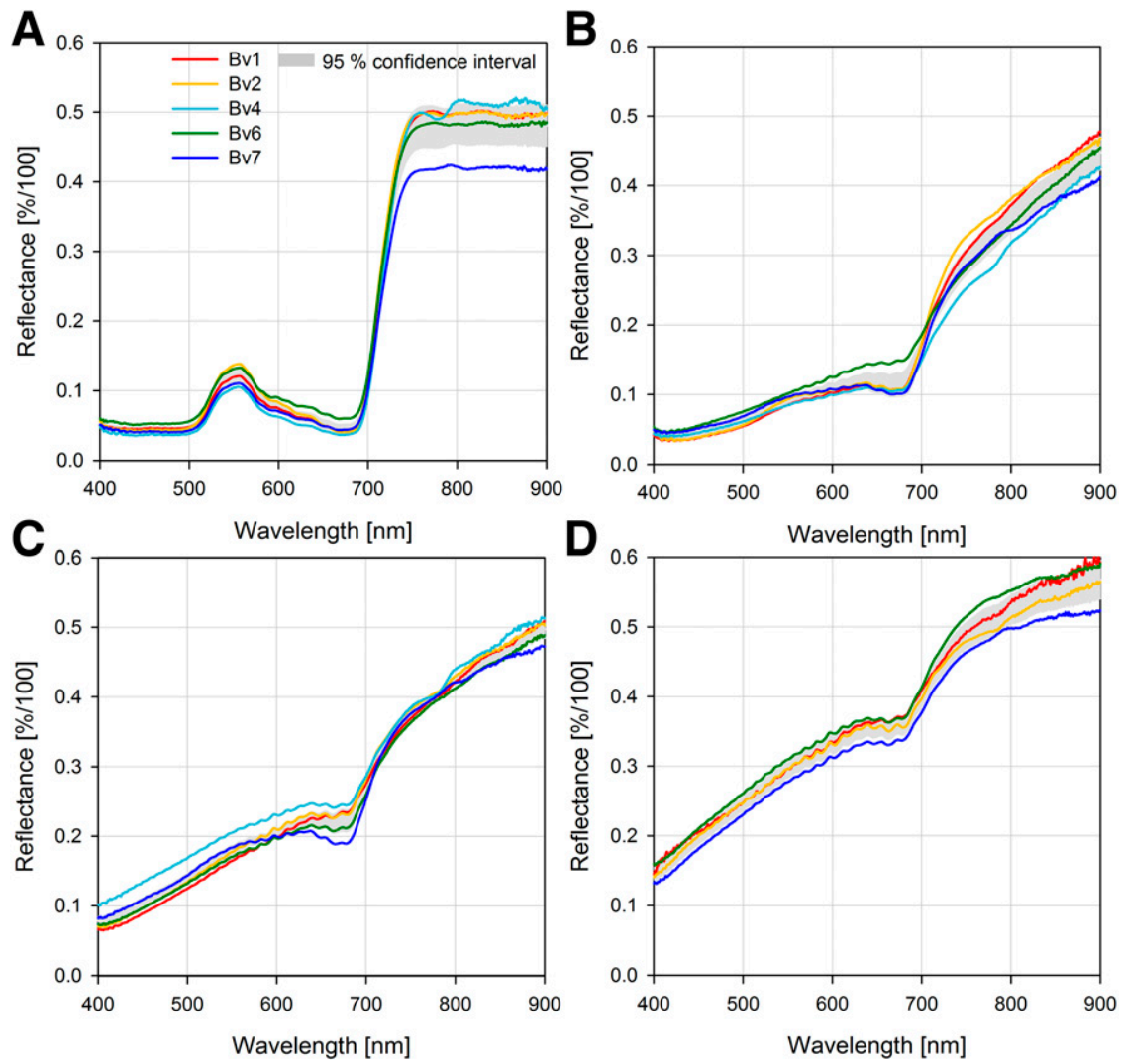

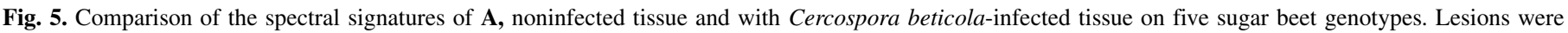

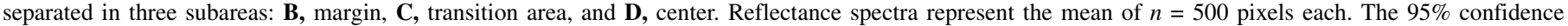
intervals for the mean of all genotypes are highlighted in gray. 

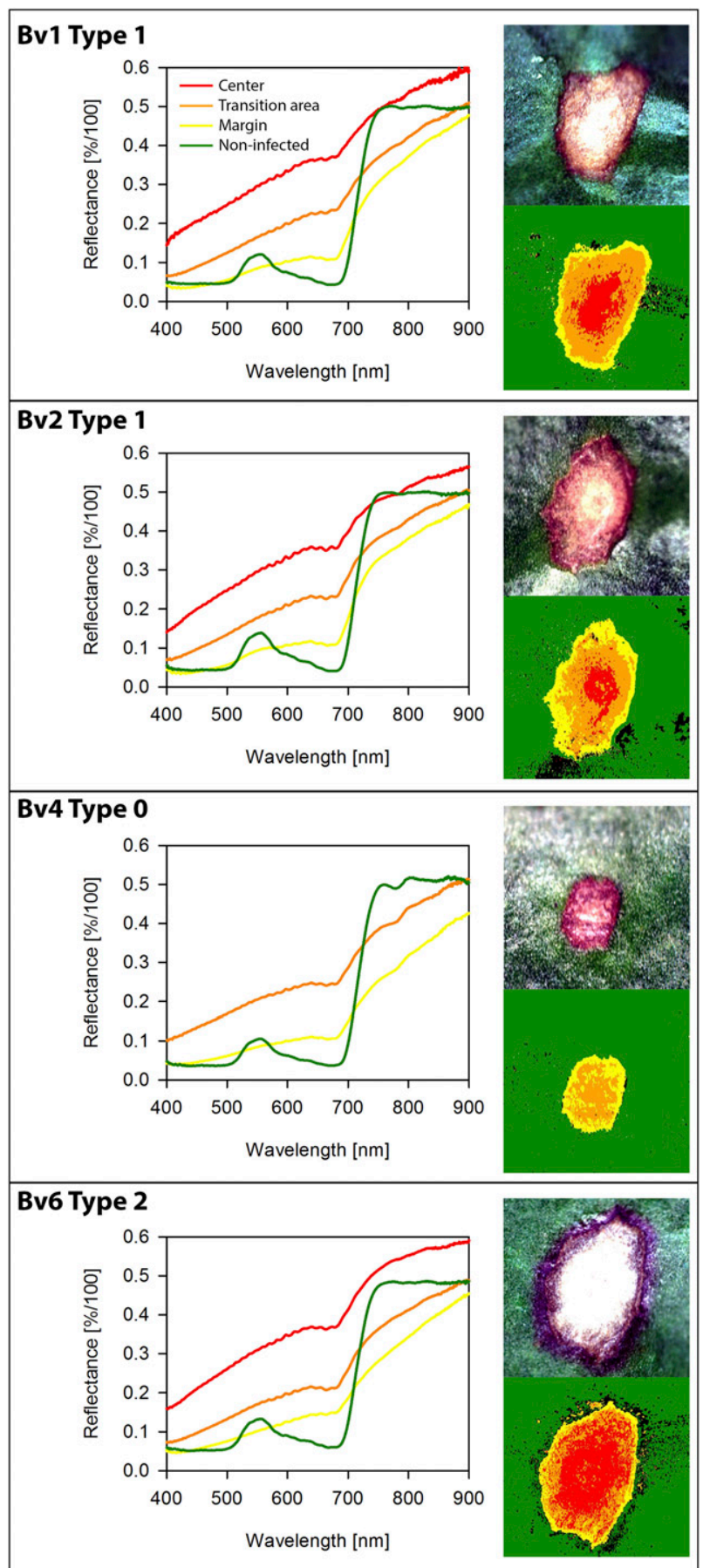

\section{Bv7 Type 2}
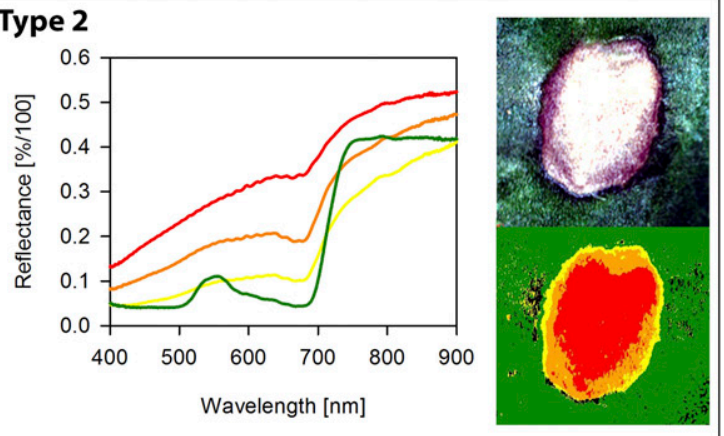

Fig. 6. Spectral signatures of Cercospora leaf spot (CLS) lesion subareas (left) and spectral angle mapper (SAM) classification based on these reference spectra. RGB images of CLS lesions of indicated class (upper right panels) and pseudo color image of SAM classification (lower right panels) for sugar beet genotypes Bv1, Bv2, Bv4, Bv6, and Bv7. Black pixels are not classified. reflectance in the VIS range was particularly characteristic for the transition area and the center, whereas the differences in the margin specific signature were more pronounced in the decrease of the NIR reflectance shoulder. Kuska et al. (2015) have shown that the visual light reflectance of barley leaves increased as powdery mildew colonies develop. A decrease of the NIR reflectance at $800 \mathrm{~nm}$ was also reported for the chocolate spot disease on Vicia faba caused by the necrotrophic fungus Botrytis fabae (Malthus and Madeira 1993). Previous studies on hyperspectral imaging of disease symptoms on sugar beet have reported the same specific reflectance spectra of CLS centers and margins without focusing on the host genotype (Mahlein et al. 2012). They described the continuously changing reflectance along transects through lesions and over time. Based on the specific signatures, lesions on sugar beet genotypes differing in CLS resistance could be differentiated into up to three subareas and the spatial proportions could be quantified. The composition of the CLS lesions, visualized by SAM classification was related to the resistance of the genotype.

Bv1

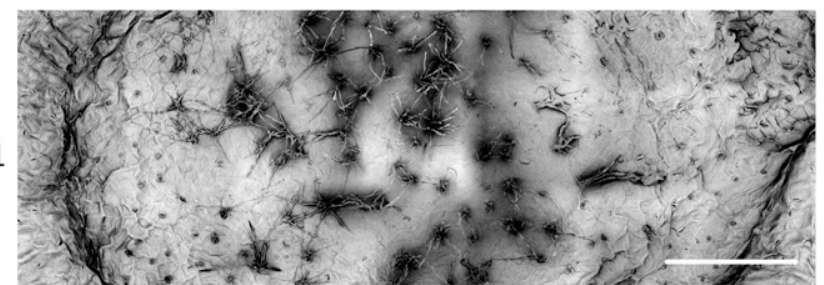

Bv2

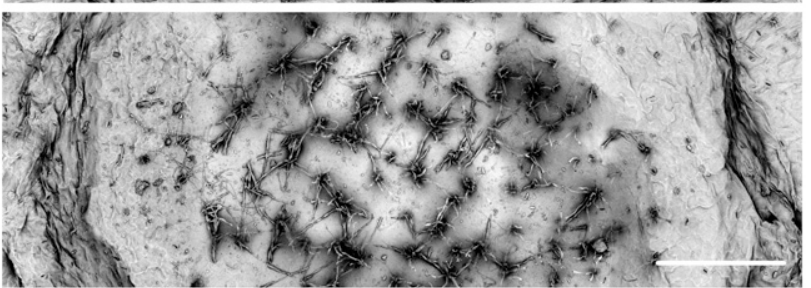

Bv4

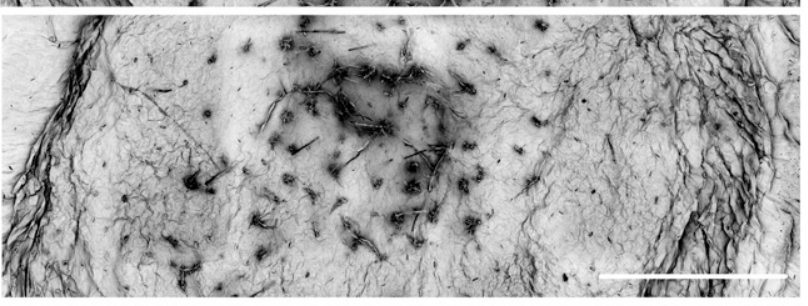

Bv6

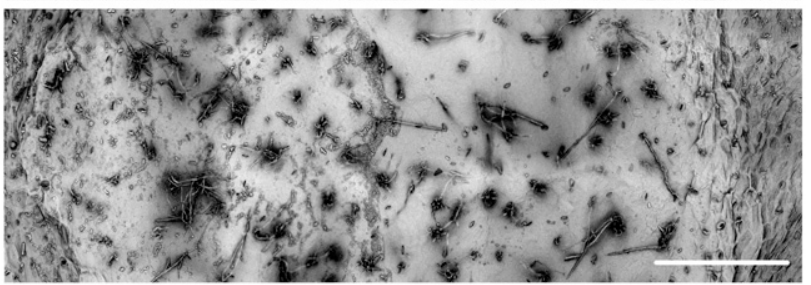

Bv7

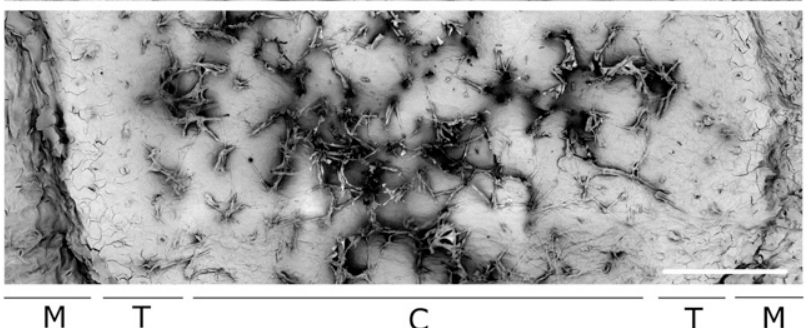

Fig. 7. Topography of Cercospora leaf spot lesions after $48 \mathrm{~h}$ incubation at $100 \%$ relative humidity 23 days after inoculation on different sugar beet genotypes. One representative lesion out of 20 replicates per genotype each is shown and margin $(\mathrm{M})$, transition $(\mathrm{T})$, and central area with conidiophores (C) are indicated at the bottom. Magnification of single scanning electron micrographs was $500 x$, which were automatically mapped to one image. Scale bars represent $400 \mu \mathrm{m}$. 
Results from microscopic investigations focusing on the cellular structure support the differentiation of lesion subareas. Steinkamp et al. (1979) described three different areas in transmission electron microscopic studies of $C$. beticola lesions. The necrotic center was characterized by many hyphae and callose-like cell wall thickenings. According to Steinkamp et al. (1979), all cells within this area had collapsed and were necrotic, thus photosynthetic pigments had been degraded and so the absorbance of visible light increased. The degree of tissue degradation and the availability of nutrients for the pathogen may influence fungal growth and sporulation, thus possibly explaining the limitation of conidiophores to the center shown in the scanning electron micrographs. $C$. beticola produces toxins in order to damage and colonize the host tissue (Daub and Ehrenshaft 2000). The photosensitizer cercosporin, for example, is an essential pathogenicity factor of $C$. beticola (Staerkel et al. 2013). The reduced lesion center size on resistant sugar beets may be due to a higher tolerance to this pathotoxin.

Furthermore, a boundary zone separating the diseased tissue from healthy leaf tissue has been identified (Steinkamp et al. 1979). This boundary zone was divided into an inner and outer region. The inner zone was characterized by necrotic cells with thickenings of the inner surface of their cell walls. Fungal hyphae invaded this region, but not the outer zone, where electron-dense material was present in the intercellular space. The two characteristic zones may correspond to the transition area and the margin as identified spectrally in this study. Pathogen growth is limited in this area which may result from active defense by the plant or decreased availability of nutrients. The transition area where the pathogen produces less and partially immature conidiophores resembles the inner boundary zone where only some hyphae were present in EM studies (Steinkamp et al. 1979). The shriveled tissue, seen in the topography of the lesions, which separates the lesions from the apparently healthy tissue is probably linked to the margin with decreased reflectance in the range from 700 to $900 \mathrm{~nm}$. The high reflectance in the NIR range is mainly caused by direct reflection on the leaf surface and scattering processes in the mesophyll (Jacquemoud and Ustin 2001), which is presumably reduced in the margin due to the shriveling of the tissue and decreased internal reflectance. This contraction of the leaf tissue in the outer area is more severe in sporulating leaf spots. So it is also conceivable that the spectral changes were influenced by extracellular material found in this area (Feindt et al. 1981; Steinkamp et al. 1979).

Interestingly, Feindt et al. (1981) hypothesized that the boundary zone of CLS lesions on susceptible sugar beets resemble the complete infection site on resistant genotypes. This is consistent with the finding that resistant genotypes had a considerably higher portion of lesions with no differentiation into center and margin than susceptible genotypes. The prevailing symptom phenotype on sugar beet genotype Bv4 lacks the SAM class center. This could also explain why the average CLS signature on Bv4 differed from that of the other genotypes. This reflectance spectrum was similar to the spectral signature of sugar beet leaves affected with $C$. beticola at a disease severity of $20 \%$, which was recorded with a nonimaging spectrometer (Mahlein et al. 2010). The reflectance curves of the CLS on the other genotypes resemble the spectral signature of leaves with increased disease severity. It remains to be determined whether the reactions in the tissue of the lesion margin play a role in pathogen defense.

Hyperspectral microscopy has the potential to categorize host plant resistance to plant pathogens and for identification of not only qualitative effects, but also quantitative differences in the frequency of lesion phenotypes as related to host resistance. Spectral information from the NIR range may be used to characterize stress-induced modifications in the structure of plant tissue, such as collapse of cells. This effect appears during pathogen colonization in addition to changes in plant pigments, influencing the visible range of the electromagnetic spectrum. Differentiation of lesion types based on subareas is improved by analyzing spectral signatures and has the potential to accelerate the screening process in breeding for CLS resistance. It offers possibilities for objective and automated phenotyping without the necessity of running time- and cost-intensive field experiments that reveal rate-reducing resistance only in the later stages of disease epidemics.

\section{ACKNOWLEDGMENTS}

We thank KWS SAAT SE (Einbeck, Germany) and W. Beyer for providing plant material and valuable support. This project is supported by funds of the Federal Ministry of Food and Agriculture (BMEL) based on a decision of the Parliament of the Federal Republic of Germany via the Federal Office for Agriculture and Food (BLE) under the innovation support program.

\section{LITERATURE CITED}

Bleiholder, H., and Weltzien, H. C. 1972. Beitraege zur Epidemiologie von Cercospora beticola Sacc. an Zuckerruebe. J. Phytopathol. 73:93-114.

Bock, C. H., Poole, G. H., Parker, P. E., and Gottwald, T. R. 2010. Plant disease severity estimated visually, by digital photography and image analysis, and by hyperspectral imaging. Crit. Rev. Plant Sci. 29:59-107.

Daub, M. E., and Ehrenshaft, M. 2000. The photoactivated Cercospora toxin cercosporin: Contributions to plant disease and fundamental biology. Annu. Rev. Phytopathol. 38:461-490.

Duffus, J. E., and Ruppel, E. G. 1993. Diseases. Pages 346-427 in: The Sugarbeet Crop. D. A. Cooke and R. K. Scott, eds. Chapman and Hall, London.

Feindt, F., Mendgen, K., and Heitefuss, R. 1980. Feinstruktur unterschiedlicher Zellwandreaktionen im Blattparenchym anfaelliger und resistenter Rueben (Beta vulgaris L.) nach Infektion durch Cercospora beticola Sacc. Phytopathol. Z. 101:248-264.

Feindt, F., Mendgen, K., and Heitefuss, R. 1981. Der Einfluss der Spaltoeffnungsweite und des Blattalters auf den Infektionserfolg von Cercospora beticola bei Zuckerrueben (Beta vulgaris L.) unterschiedlicher Anfaelligkeit. Phytopathol. Z. 101:281-297.

Fetch, T. G., and Steffenson, B. J. 1999. Rating scales for assessing infection responses of barley infected with Cochliobolus sativus. Plant Dis. 83:213-217.

Foster, D. J., Beute, M. K., and Wynne, J. C. 1980. Spore production and latent period as mechanisms of resistance to Cercospora arachidicola in four peanut genotypes. Peanut Sci. 7:88-90.

Gottschalk, T. E., Mikkelsen, J. D., Nielsen, J. E., Nielsen, K. K., and Brunstedt, J. 1998. Immunolocalization and characterization of a $\beta-1,3-$ glucanase from sugar beet, deduction of its primary structure and nucleotide sequence by cDNA and genomic cloning. Plant Sci. 132:153-167.

Goudet, C., Milat, M. L., Sentenac, H., and Thibaud, J. B. 2000. Beticolins, nonpeptidic, polycyclic molecules produced by the phytopathogenic fungus Cercospora beticola, as a new family of ion channel-forming toxins. Mol. Plant-Microbe Interact. 13:203-209.

Holtschulte, B. 2000. Cercospora beticola-worldwide distribution and incidence. Pages 5-16 in: Cercospora beticola Sacc. Biology, Agronomic Influence and Control Measures in Sugar Beet 2000, Vol. 2. Advances in Sugar Beet Research. M. J. C. Asher, B. Holtschulte, M. R. Molard, F. Rosso, G. Steinrucken, and R. Beckers, eds. International Institute for Beet Research, Brussels, Belgium.

Jacquemoud, S., and Ustin, S. L. 2001. Leaf optical properties: A state of the art. Pages 223-332 in: 8th International Symposium of Physical Measurements \& Signatures in Remote Sensing, CNES, Aussois, France.

Jensen, J. R. 2007. Remote Sensing of the Environment: An Earth Resource Perspective. 2nd ed. K. C. Clarke, ed. Prentice Hall, New York.

Karadimos, D. A., Karaoglanidis, G. S., and Tzavella-Klonari, K. 2005. Biological activity and physical modes of action of the Qo inhibitor fungicides trifloxystrobin and pyraclostrobin against Cercospora beticola. Crop Prot. 24:23-29.

Karaoglanidis, G. S., Ioannidis, P. M., and Thanassoulopoulos, C. C. 2000. Reduced sensitivity of Cercospora beticola isolates to sterol demethylation inhibiting fungicides. Plant Pathol. 49:567-572.

Kirk, W. W., Hanson, L. E., Franc, G. D., Stump, W. L., Gachango, E., Clark, G., and Stewart, J. 2012. First report of strobilurin resistance in Cercospora beticola in sugar beet (Beta vulgaris) in Michigan and Nebraska, USA. New Dis. Rep. 26:3.

Kolmer, J. A. 1996. Genetics of resistance to wheat leaf rust. Annu. Rev. Phytopathol. 34:435-455.

Kuska, M., Wahabzada, M., Leucker, M., Dehne, H.-W., Kersting, K., Oerke, E.-C., Steiner, U., and Mahlein, A.-K. 2015. Hyperspectral phenotyping on 
the microscopic scale: Towards automated characterization of plant-pathogen interactions. Plant Methods 11:28.

Mahlein, A.-K., Steiner, U., Dehne, H.-W., and Oerke, E.-C. 2010. Spectral signatures of sugar beet leaves for the detection and differentiation of diseases. Precis. Agric. 11:413-431.

Mahlein, A.-K., Steiner, U., Hillnhuetter, C., Dehne, H.-W., and Oerke, E.-C. 2012. Hyperspectral imaging for small-scale analysis of symptoms caused by different sugar beet diseases. Plant Methods 8:3.

Malthus, T. J., and Madeira, A. C. 1993. High resolution spectroradiometry: Spectral reflectance of field bean leaves infected by Botrytis fabae. Remote Sens. Environ. 45:107-116.

Meier, U. 2001. Entwicklungsstadien mono- und dikotyler Pflanzen. Biol. Bundesanstalt fuer Land- und Forstwirtschaft. 2:1-165.

Montes, J. M., Melchinger, A. E., and Reif, J. C. 2007. Novel throughput phenotyping platforms in plant genetic studies. Trends Plant Sci. 12:433-436.

Mutka, A. M., and Bart, R. S. 2015. Image-based phenotyping of plant disease symptoms. Front. Plant Sci. 5:734.

Nielsen, K. K., Nielsen, J. E., Madrid, S. M., and Mikkelsen, J. D. 1997. Characterization of a new antifungal chitin-binding peptide from sugar beet leaves. Plant Physiol. 113:83-91.

Niks, R. E., and Kuiper, H. J. 1983. Histology of the relation between minor and major genes for resistance of barley to leaf rust. Phytopathology 73:55-59.

Parlevliet, J. E. 1976. The genetics of seedling resistance to leaf rust, Puccinia hordei Otth. in some spring barley cultivars. Euphytica 25:249-254.

Parlevliet, J. E. 1979. Components of resistance that reduce the rate of epidemic development. Annu. Rev. Phytopathol. 17:203-222.

Ricker, M. D., Beute, M. K., and Campbell, C. L. 1985. Components of resistance in peanut to Cercospora arachidicola. Plant Dis. 69:1059-1064.

Rossi, V., Battilani, P., Chiusa, G., Giosuè, S., Languasco, L., and Racca, P. 1999. Components of rate-reducing resistance to Cercospora leaf spot in sugar beet: Incubation length, infection efficiency, lesion size. J. Plant Pathol. 81:25-35.

Rossi, V., Battilani, P., Chiusa, G., Giosuè, S., Languasco, L., and Racca, P. 2000. Components of rate-reducing resistance to Cercospora leaf spot in sugar beet: Conidiation length, spore yield. J. Plant Pathol. 82: $125-131$.

Ruppel, E. G. 1972. Variation among isolates of Cercospora beticola from sugar beet. Phytopathology 62:134-136.

Savitzky, A., and Golay, M. J. E. 1964. Smoothing and differentiation of data by simplified least squares procedures. Anal. Chem. 36:1627-1629.

Shane, W. W., and Teng, P. S. 1992. Impact of Cercospora leaf spot on root weight, sugar yield, and purity of Beta vulgaris. Plant Dis. 76:812-820.

Smith, G. A., and Campbell, L. G. 1996. Association between resistance to Cercospora and yield in commercial sugarbeet hybrids. Plant Breed. 115:28-32.

Staerkel, C., Boenisch, M. J., Kroeger, C., Bormann, J., Schaefer, W., and Stahl, D. 2013. CbCTB2, an O-methyltransferase is essential for biosynthesis of the phytotoxin cercosporin and infection of sugar beet by Cercospora beticola. BMC Plant Biol. 13:50.

Steinkamp, M. P., Martin, S. S., Hoefert, L. L., and Ruppel, E. G. 1979. Ultrastructure of lesions produced by Cercospora beficola in leaves of Beta vulgaris. Physiol. Plant Pathol. 15:13-26.

Trkulja, N., Ivanović, Ž., Pfaf-Dolovac, E., Dolovac, N., Mitrović, M., Toševski, I., and Jović, J. 2012. Characterisation of benzimidazole resistance of Cercospora beticola in Serbia using PCR-based detection of resistance-associated mutations of the $\beta$-tubulin gene. Eur. J. Plant Pathol. 135:889-902.

Weiland, J., and Koch, G. 2004. Sugarbeet leaf spot disease (Cercospora beticola Sacc.). Mol. Plant Pathol. 5:157-166.

Weltmeier, F., Mäser, A., Menze, A., Hennig, S., Schad, M., Breuer, F., Schulz, B., Holtschulte, B., Nehls, R., and Stahl, D. J. 2011. Transcript profiles in sugar beet genotypes uncover timing and strength of defense reactions to Cercospora beticola infection. Mol. Plant-Microbe Interact. 24: 758-772.

Wolf, P., and Verreet, J. 2002. The IPM sugar beet model. Plant Dis. 86:336-344.

Yuhas, R., Goetz, F. H., and Boardman, J. W. 1992. Discrimination among semi-arid landscape endmembers using the Spectral Angle Mapper (SAM) algorithm. Summaries of the Third Annual JPL Airborne Geoscience Workshop. Jet Propulsion Laboratory Publication 92:147-149. 